\title{
Experimental infrared measurements for hydrocarbon pollutant determination in subterranean waters
}

\author{
A. Lay-Ekuakille, ${ }^{1, \text { a) }}$ I. Palamara, ${ }^{2}$ D. Caratelli, ${ }^{3}$ and F. C. Morabito ${ }^{2}$ \\ ${ }^{1}$ Department of Innovation Engineering, University of Salento, 73100 Lecce, Italy \\ ${ }^{2}$ Department of Mechanics and Materials, NeuroLab, University "Mediterranean" of Reggio C., \\ 89124 Reggio C., Italy \\ ${ }^{3}$ Microwave Technology and Systems for Radar, Delft University of Technology, 2628 CD Delft, \\ The Netherlands
}

(Received 12 August 2012; accepted 3 December 2012; published online 4 January 2013)

\begin{abstract}
Subterranean waters are often polluted by industrial and anthropic effluents that are drained in subsoil. To prevent and control pollution, legislations of different developed countries require an online monitoring measurement, especially for detecting organic solvents (chlorinated and unchlorinated ones). Online measurements include both real-time and no real-time measurements. In general, it is difficult to implement real-time measurements in stricto sensu for online acquisitions on aqueous effluents since they need to be processed by a modeling. This research presents an experimental measurement system based on infrared (IR) spectroscopy for aqueous effluents containing hydrocarbons and capable of displaying excellent values of pollutant concentrations even in instable conditions; the system is able to detect pollutants either in laminar or turbulent flow. The results show the possibility of avoiding the use of "Pitot tube" that is employed to create a stagnation point in order to convert kinetic energy into potential one. This conversion allows the transformation of a turbulent flow in a laminar flow making easy measurement of pollutants included in an aqueous effluent. Obviously, "Pitot tube" is also used for other fluid effluents. The obtained results have been compared with those produced by means of sophisticated IR instrumentation for laboratory applications. (0) 2013 American Institute of Physics. [http://dx.doi.org/10.1063/1.4772576]
\end{abstract}

\section{INTRODUCTION}

Many of the major problems that humanity is facing in the 21 st century are related to water quantity and/or water quality issues. ${ }^{1}$ These problems are going to be more aggravated in the future by climate change, resulting in higher water temperatures, melting of glaciers, and an intensification of the water cycle, ${ }^{2}$ with potentially more floods and droughts. ${ }^{3}$ With respect to human health, the most direct and most deep impact is the lack of improved sanitation, and related to it is the lack of safe drinking water, which currently affects more than a third of the people in the world. Additional threats include, for example, exposure to pathogens or to chemical toxicants via the food chain (e.g., the result of irrigating plants with contaminated water and of bioaccumulation of toxic chemicals by aquatic organisms, including seafood and fish) or during recreation (e.g., swimming in polluted surface water). This paper deals with the characterization of groundwater pollution. Surface water and groundwater have often been studied and managed as separate resources, although they are interrelated. ${ }^{4}$ Surface water seeps through the soil and becomes groundwater. Conversely, groundwater can also feed surface water sources. Sources of surface water pollution are generally grouped into two categories based on their origin. Point sources refer to contaminants that sink a waterway from a single, identifiable source, such as a pipe

\footnotetext{
a) Author to whom correspondence should be addressed. Electronic mail: aime.lay.ekuakille@unisalento.it. Telephone: +39.0832.297822. Fax +39.0832.297827
}

or a ditch. Examples of sources in this category include discharges from sewage treatment plant, a factory or a city storm drain. Nonpoint source pollution refers to diffused contamination that does not originate from a single discrete source. The majority of water pollutants is eventually carried into the oceans by rivers. The influence can be traced hundred miles from the origin by studies using hydrology transport models. More than one-third of Earth's accessible renewable freshwater is consumptively used for agricultural, industrial, and domestic purposes. ${ }^{5}$ As most of these activities lead to water contamination with diverse synthetic and natural chemicals, it comes as no surprise that chemical pollution of natural water has become a major public concern in almost all parts of the world. Particularly, our interest is on hydrocarbon pollution that can produce serious health disorders like respiratory diseases, heart diseases, cancer, and even death. Hydrocarbon pollution may be analyzed by several categories of methods: physical, chemical, and biological. Many involve collection of samples, followed by specialized analytical tests. In this paper, we propose a detection method of pollution that may be conducted in situ, without sampling. This research presents an experimental measurement system based on infrared (IR) spectroscopy for aqueous effluents containing hydrocarbons. The paper is organized as follows. In Sec. II, light issues are recalled for absorption and scattering problems. Section III illustrates materials and methods related to the experimental setup. Results and discussion are depicted in Sec. IV while validation through commercial and sophisticated instrumentation is explained in Sec. V. The last one deals with the final outlook of the paper. 


\section{LIGHT TRANSMISSION: AN OVERVIEW}

Light transmission through a water sample is determined by physical properties such as particle size, shape, composition, and chemical properties. There is enormous variation in these properties in the environment, resulting in a nearly infinite number of unique optical characteristics for natural and man-influenced water, however, consistent light transmission through a water sample is essential for precise measurements. Light transfer through a water sample is affected in complex ways by water molecules, material dissolved in the water, and scattering by suspended particles. ${ }^{6}$

\section{A. Absorption}

Absorption represents the reduction of the total irradiance incident beam caused by the energy being converted into another form. Along with scattering this accounts for the total attenuation of all the coming radiation caused by water body. ${ }^{7}$ Currently one of the most common absorption methodology is infrared spectroscopy. Simply, it is the absorption measurement of different IR frequencies by a sample positioned in the path of an incident beam. Examination of the transmitted light reveals how much energy was absorbed at each frequency (or wavelength). IR absorption information is generally presented in the form of a spectrum with wavelength or wavenumber as the $\mathrm{x}$-axis and absorption intensity or transmittance as the yaxis. The absorption coefficient here indicated as $k(v)$ is defined as the fractional decrease in the flux density at frequency $v$ per unit path length through the absorbing medium

$$
-\delta I(v)=I(v) k(v) \delta x,
$$

where $I(v)$ is the flux density incident on the layer of thickness $\delta(x)$. The units of $k$ are reciprocal length, or $\mathrm{m}^{-1}$. For a homogenous layer of thickness $l$ this equation can be integrated to give

$$
I_{l}(v)=I_{0}(v) e^{-k(v) l} .
$$

The product $k(v) l$ defines the optical depth $\tau(\nu)$ at frequency $v$. If the absorbing medium is not homogenous,

$$
\tau(v)=\int_{0}^{l} k(v) d v .
$$

The absorption from an isolated spectral line is spread over a finite frequency range. The absorption coefficient can be easily related to Einstein coefficient ${ }^{8}$ by considering the total power absorbed from the incident beam. Integrating Eq. (1) over the lines gives

$$
-\Delta I=I\left(v_{0}\right) \delta x \int_{\text {line }} k(v) d v \text { in } \mathrm{Wm}^{-2} .
$$

\section{B. Scattering}

Light scattering changes the direction of photon transport, "dispersing" them as they penetrate a sample. Scattering occurs when electromagnetic radiation (light) interacts with matter; the oscillating electric field induces oscillations in the charges of the molecules and atoms in the material. These oscillating dipoles re-radiate secondary (scattered) radiation in all directions. In a certain direction at a distant observation point, the total scattered field is the superposition of all the emitted radiation from all the dipoles in the particle. When there is no change in wavelength between the incident and scattered radiation, it is known as elastic scattering. Any interaction which results in a change in the wavelength of the scattered light relative to the incident is known as inelastic scattering. ${ }^{7}$ The theory of light scattering by particles is well presented in Ref. 9. The two most important inelastic processes in natural waters are fluorescence (by chlorophyll) and Raman scattering (by waters molecules). Both fluorescence and Raman scattering occur when a molecule absorbs an incident photon then emits a photon at a longer wavelength (fluorescence) or shorter wavelength (Raman scattering).

The scattering behaviour depends on the properties of the light as well as those of the particles. In a perfectly homogenous and isotropic medium, the scattered radiation medium interferes destructively, so that there is no scattered radiation. In real materials density fluctuations and/or refractive index variations always lead to some scattering. The scattering properties of the particles are characterized by their sizes, shapes, orientation, and refractive indices. There are several theoretical approaches, both analytic and numerical, for calculating scattering properties, the simplest of which is the Rayleigh scattering.

The intensity, $I$, of the scattered radiation is given by

$$
I=I_{0}\left(\frac{1+\cos ^{2} \theta}{2 R^{2}}\right)\left(\frac{2 \pi}{\lambda}\right)^{4}\left(\frac{n^{2}-1}{n^{2}+2}\right)^{2}\left(\frac{d}{2}\right)^{6},
$$

where $I_{0}$ is the light intensity before the interaction with the particle, $R$ is the distance between the particle and the observer, $\theta$ is the scattering angle, $n$ is the refractive index of the particle, and $d$ is the diameter of the particle.

It can be seen from the above equation that Rayleigh scattering is strongly dependent upon the size of the particle and the wavelengths. The intensity of the Rayleigh scattered radiation increases rapidly as the ratio of particle size to wavelength increases. Furthermore, the intensity of Rayleigh scattered radiation is identical in the forward and reverse directions. $^{9}$

The Rayleigh scattering model is not useful when the particle size becomes larger than about $10 \%$ of the wavelength of the incident radiation. In the case of particles with dimensions greater than this, Mie's scattering model can be used to find the intensity of the scattered radiation. The intensity of Mie scattered radiation is given by the summation of an infinite series of terms rather than by a simple mathematical expression.

\section{MATERIALS AND METHODS FOR EXPERIMENTAL SETUP}

\section{A. Materials}

Samples used, in the experiment described in this paper, are collected from sampling facilities installed in appropriate and dedicated wells realized around an industrial area in order to monitor pollutants discharged in subterranean matrices in unintentional way. The above monitoring wells are 
also used as counter-check to avoid contamination from other plants like landfills, ground irrigation with prohibited products, etc. The samples contain water with hydrocarbon pollutants at different concentrations. The hydrocarbons, in general, come from industrial drain and runoff water. The latter case is related to the presence of trucks (for example) in a parking area. However, even the parking area is paved, leaks of water containing hydrocarbons (especially fuels) can reach ground, soil, etc. All samples are given by Italian National Institute for Micro-Pollutants (INCA) and their pollutant concentration has been determined using gravimetric analysis. All gravimetric analyses rely on some final determination of weight as a means of quantifying an analyte. This kind of analysis is potentially one of the most accurate classes of analytical methods available. The ideal product of a gravimetric analysis should be pure, insoluble, and easy filterable. These requirements are not easy to meet and in our case particles can pass through filter, for this reason appropriate techniques to promote particles growth are used.

\section{B. Experimental setup}

The used experimental setup is shown in Fig. 1(a). Due to the different size of the hydrocarbon microparticles a broad white lamp source (Halogen light source HL-2000-FHSA Mikropack) is used; in this manner, it is possible to observe a "macroscopic" effect because a lot of wavelength will interact with the microparticles. The lamp source is coupled to a multimode optical fiber (optical fiber source see Table I) fixed on a holder micrometric stage. At the same height of the optical fiber source is fixed an optical fiber receiver directly connected to a high sensitive optical multichannel analyzer (OMA). We observe that the distances $\mathrm{d} 1$ and $\mathrm{d} 2$ of Fig. 1(b) are tuned in order to improve a sufficient light collected by the receiver fiber. Due to the high sensitivity of the OMA and to the big dimension of the sample we do not use a telescope system as used for integrated optical waveguides or bulk type materials, where a good collimated light is required. ${ }^{10,11}$ The pollution of a liquid is often characterized by floating microparticles. Due to the dimensions, these microparticles interact with the light: the light coming from an external source can be absorbed and scattered inside the not pure liquid. We use this basic principle in order to detect, in real time, the pollution of a water sample with hydrocarbons. Figure 2(a) shows the case of a light source ${ }^{12,13}$ which excites a fixed sample (without motion): due to the stable condition, low quantities of microparticles float inside the water and, consecutively, a big quantity of the light will be transmitted at the output of the sample (corresponding to a low quantity of ab- (a)

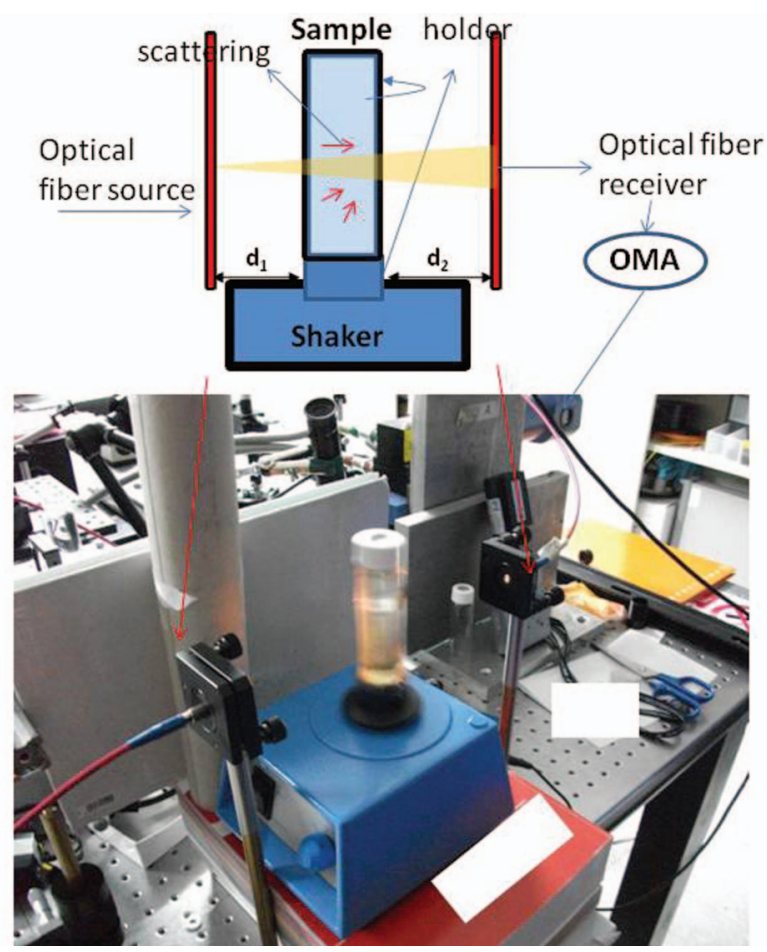

FIG. 1. (a) Schematic illustration of the experimental setup. The light source region is defined by the light cone. (b) Photo of the used experimental setup.

sorbed light of the liquid). By applying a shaker to the sample a big quantity of initially deposited hydrocarbon microparticles will be uniformly dispersed in all the volume of the sample, and consecutively, a bigger quantity of the light will be absorbed, by decreasing the transmitted light at the output of the sample (Fig. 2(b)).

\section{MEASUREMENTS: RESULTS AND DISCUSSION}

According to the experimental setup, we characterized different samples with different concentration of hydrocarbon. The kinds of hydrocarbon are $\mathrm{C}_{14}$ and $\mathrm{C}_{40}$, respectively. Samples are labeled with a specific code. As it is shown in Table II, each sample has a different concentration. Concentrations of hydrocarbons, as validation, have been determined by means of mass spectrometer mod. 5974 Agilent and chromatographic gas 6890 Agilent; both instruments are illustrated in Fig. 3. ${ }^{14}$

When a beam of light passes through a suspension, an incident electromagnetic radiation interacts with the suspended particles and part of it gets dissipated due to absorption, reflection, and refraction, giving a turbid appearance to the

TABLE I. Datasheet of the optical Tx/Rx probes Item FT 200 EMT-visible to near-IR transmission (low OH).

\begin{tabular}{lccc}
\hline \hline Core Diameter & Cladding diameter & Coating diameter & Numerical aperture \\
\hline $200 \pm 5 \mu \mathrm{m}$ & $225 \pm 5 \mu \mathrm{m}$ & $500 \pm 30 \mu \mathrm{m}$ & $0.39 \pm 0.02$ \\
Maximum attenuation & Maximum core offset & Bend radius short/long term & Stripping tool \\
$6 \mathrm{~dB} / \mathrm{km}$ & $5 \mu \mathrm{m}$ & $6 \mathrm{~mm} / 12 \mathrm{~mm}$ & $\mathrm{~T} 12 \mathrm{~S} 21$ \\
\hline \hline
\end{tabular}

${ }^{\mathrm{a} B a s e d}$ on $5 \mathrm{GW} / \mathrm{cm}^{2}$ for $1064 \mathrm{~nm} \mathrm{Nd}$ :YAG laser with $10 \mathrm{nsec}$ pulse length and input spot size equal to $80 \%$ of the core diameter.

${ }^{\mathrm{b}}$ Based on $1 \mathrm{MW} / \mathrm{cm}^{2}$ for $1064 \mathrm{~nm} \mathrm{Nd}$ :YAG laser and input spot size equal to $80 \%$ of the core diameter. 

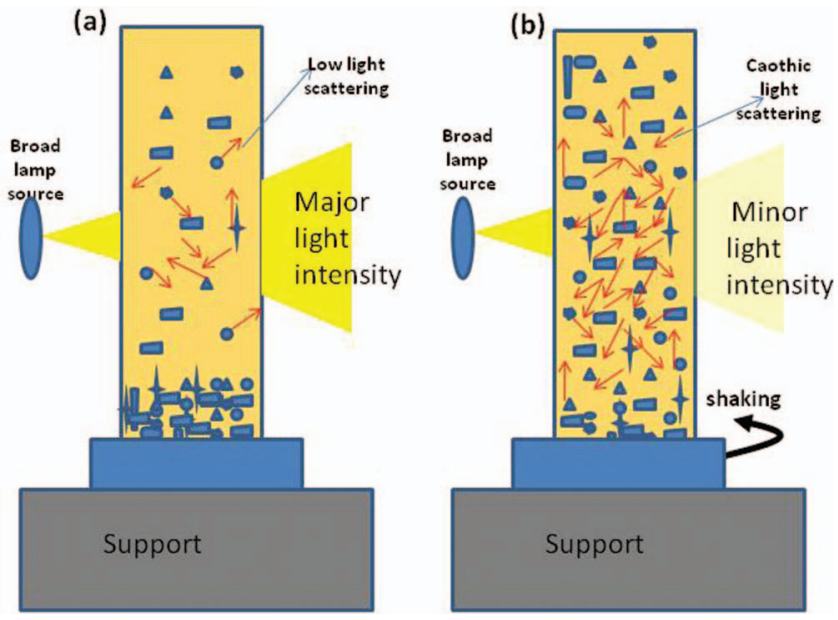

FIG. 2. (a) Light scattering of a liquid sample with hydrocarbons (without vibrations). (b) Light scattering of the vibrating sample.

mixture. The unabsorbed part of the radiant energy gets transmitted. The intensity of the transmitted radiation $I$ can be expressed as

$$
I=I_{0} 10^{\varepsilon L},
$$

where $I_{0}$ is the intensity of the incident light at a given wavelength, $I$ is the transmitted intensity, $L$ the path length, and $\varepsilon$ is a constant known as the molar absorptivity or extinction coefficient. If there are no microparticles of pollution, hence $\varepsilon=$ 0 and $I_{0}=I$.

Transmittance, $T$, can be expressed as the ratio of radiant power transmitted $(I)$ to the radiant power incident beam on the sample $\left(I_{0}\right)$

$$
T=\frac{I}{I_{0}}
$$

Absorption and transmission spectra represent equivalent information and one can be calculated from the other through a mathematical transformation. An absorption spectrum can be quantitatively related to the amount of material present using the Beer-Lambert law; it states that the absorbance of a solution is directly proportional to the concentration of the absorbing species in the solution and the path length as confirmed in Table III. This method is often used in a quantitative way to determine concentration of an absorbing species in

TABLE II. Label and concentration of samples.

\begin{tabular}{lc}
\hline \hline Label & Concentration (mg/l) \\
\hline 565 & 0.15 \\
563 & 0.2 \\
566 & 0.21 \\
570 & 0.34 \\
572 & 0.44 \\
575 & 0.53 \\
576 & 0.75 \\
580 & 1.42 \\
582 & 10.2 \\
\hline \hline
\end{tabular}

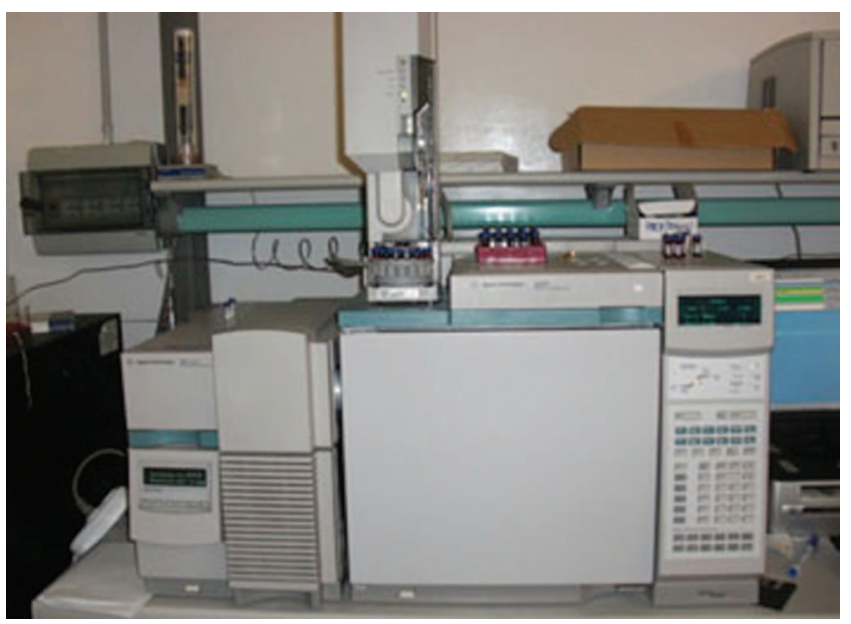

FIG. 3. Mass spectrometer and chromatographic gas for concentration determination.

solution

$$
A=-\log _{10} T=-\log _{10} \frac{I}{I_{0}}=\varepsilon \cdot c \cdot L .
$$

For a fixed path length samples for low concentrations samples the probability to find microparticles floating in all the volume increases and, consecutively, more particles will be located in the volume region corresponding the light source (see Figs. 4-7). In this last case the absorption effect of the light corresponding to the light source region is bigger, and the optical transmissivity decreases (see Fig. 8), and the absorption effect of the light corresponding to the light source region is bigger (see Fig. 9).

Transmittance and absorbance are interconnected value and using relationship (8), it is simple to determine concentration value of a substance in a sample. Increasing concentration decreases transmissivity and increases absorbance (Fig. 9). The measurements are carried out in pre-established conditions.

In order to see the influence of increasing microparticles that can float near the light source region, we apply a shaker. By considering the fixed sample 582 (without improving vibrations), we observe in Fig. 4 a low variation of the transmitted optical intensity. This variation becomes strong if we apply the shaker as illustrated in Fig. 10. In this case, the

TABLE III. Absorbance calculated with Lambert-Beer formula with $I=3$ $\mathrm{cm}$ and $\varepsilon=8355 \mathrm{~m} / \mathrm{M}$.

\begin{tabular}{lc}
\hline \hline Label & Absorbance \\
\hline 565 & 3.75 \\
563 & 5.01 \\
566 & 5.26 \\
570 & 8.5 \\
572 & 11.02 \\
575 & 13.28 \\
576 & 18.8 \\
580 & 35.6 \\
582 & 255.66 \\
\hline \hline
\end{tabular}




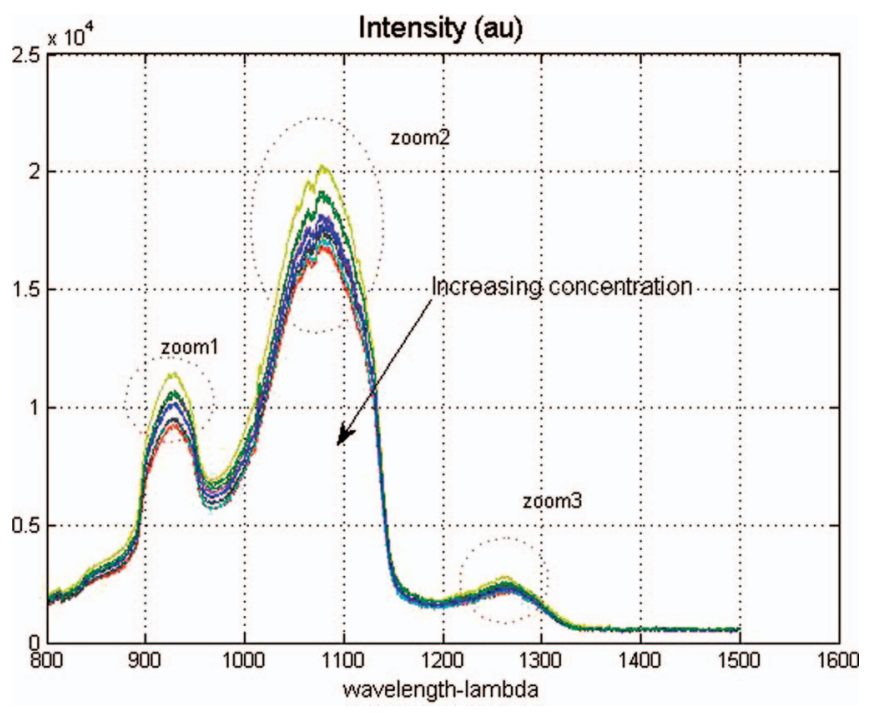

FIG. 4. Light intensity profiles for different sample concentration.

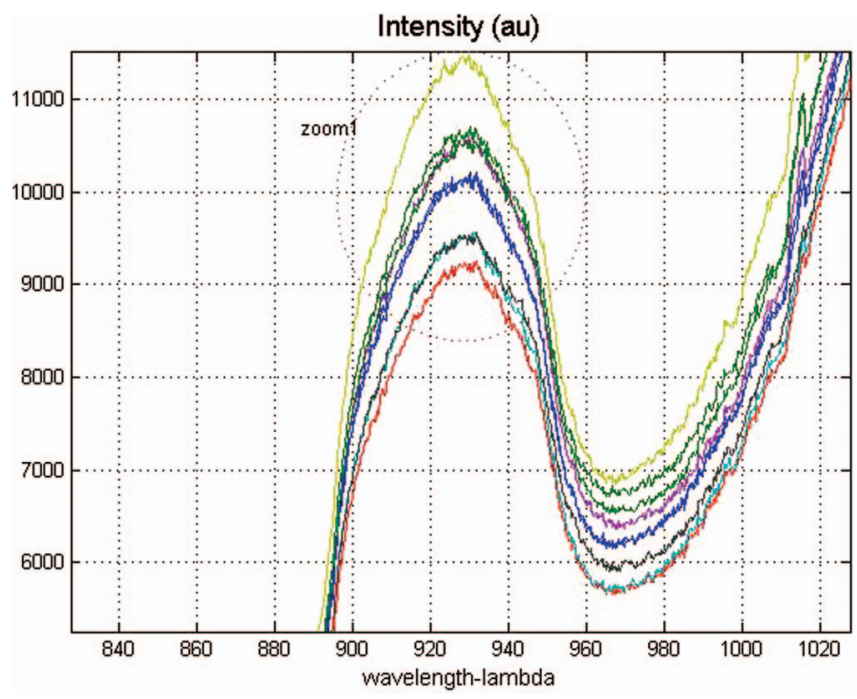

FIG. 5. Zoomed of area of interest: zoom1.

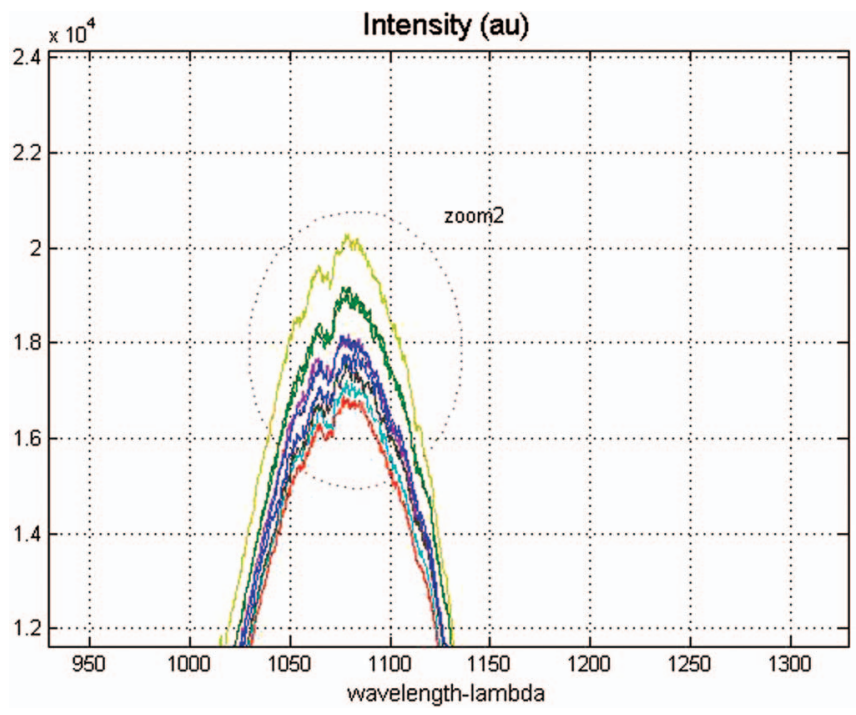

FIG. 6. Zoomed of area of interest: zoom2.

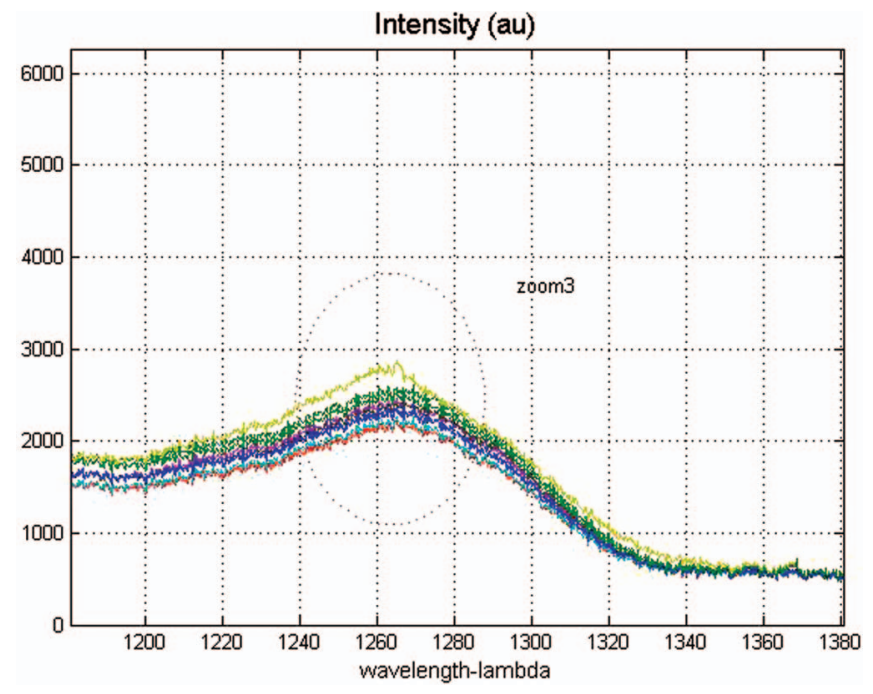

FIG. 7. Zoomed of area of interest: zoom3.

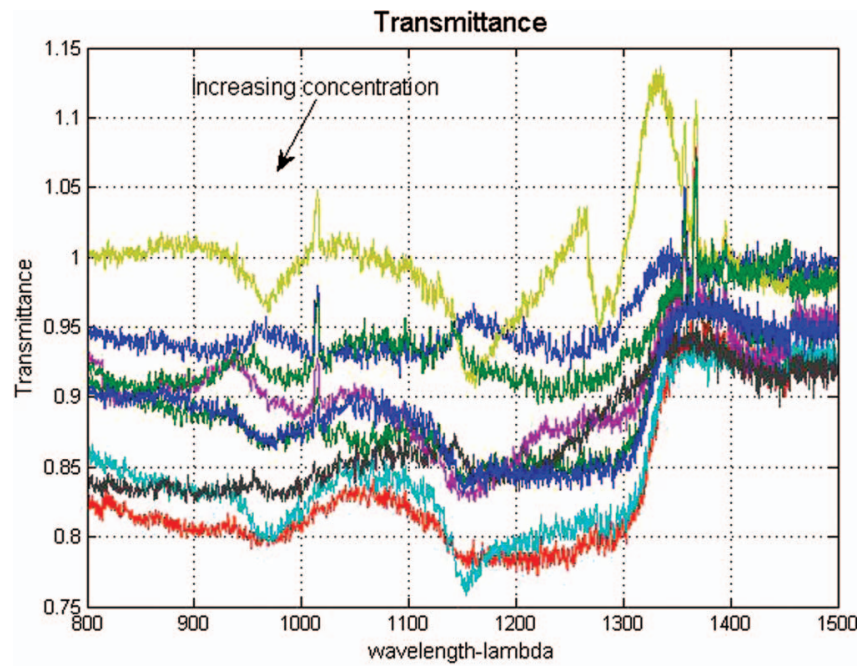

FIG. 8. Transmittance profiles for different sample concentration.

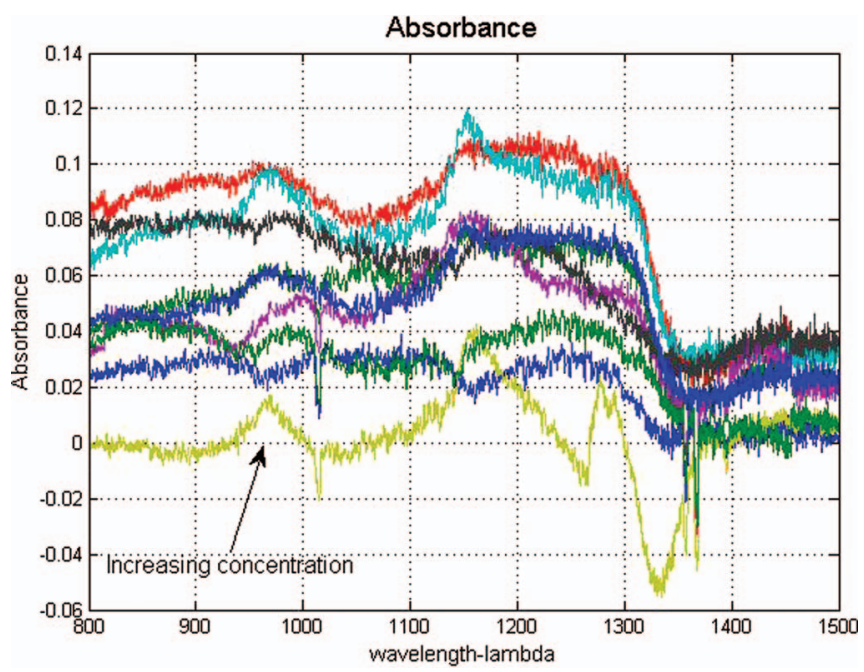

FIG. 9. Absorbance profiles for different sample concentration. 


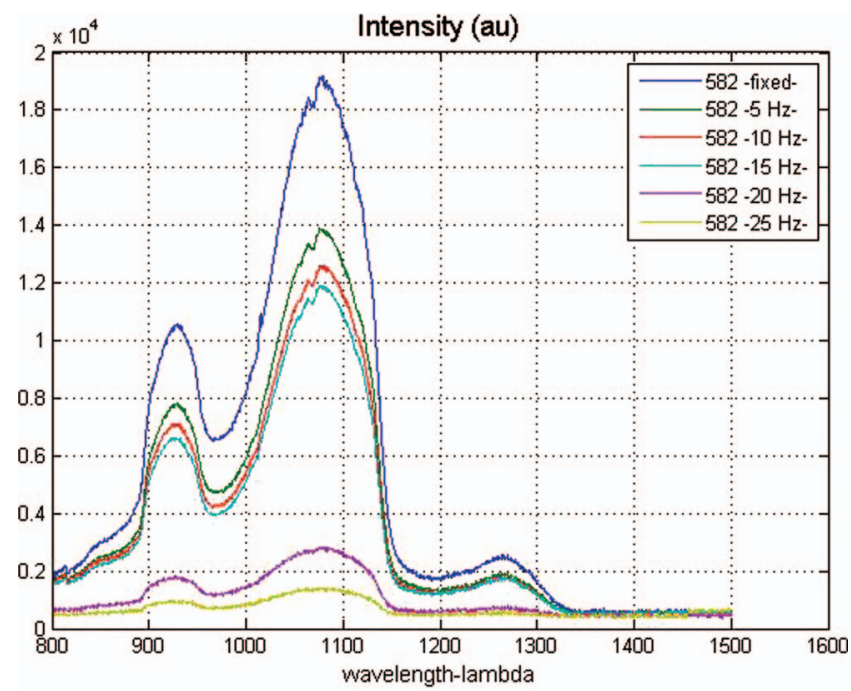

FIG. 10. Light intensity profiles for sample 582 fixed and at different speed of water flow.

\section{Transmittance}

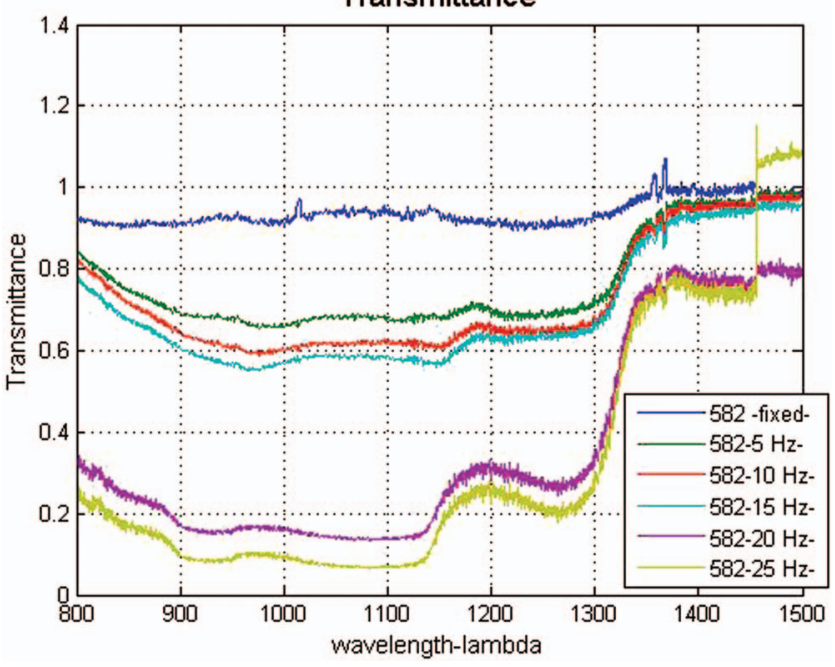

FIG. 11. Transmittance profiles for sample 582 fixed and at different speed of water flow.

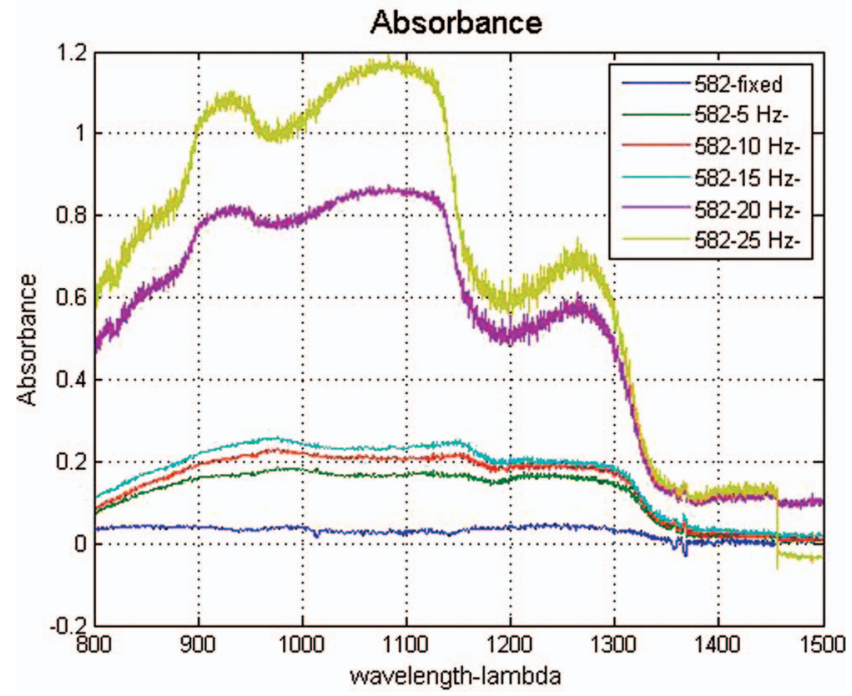

FIG. 12. Absorbance profiles for sample 582 fixed and at different speed of water flow.

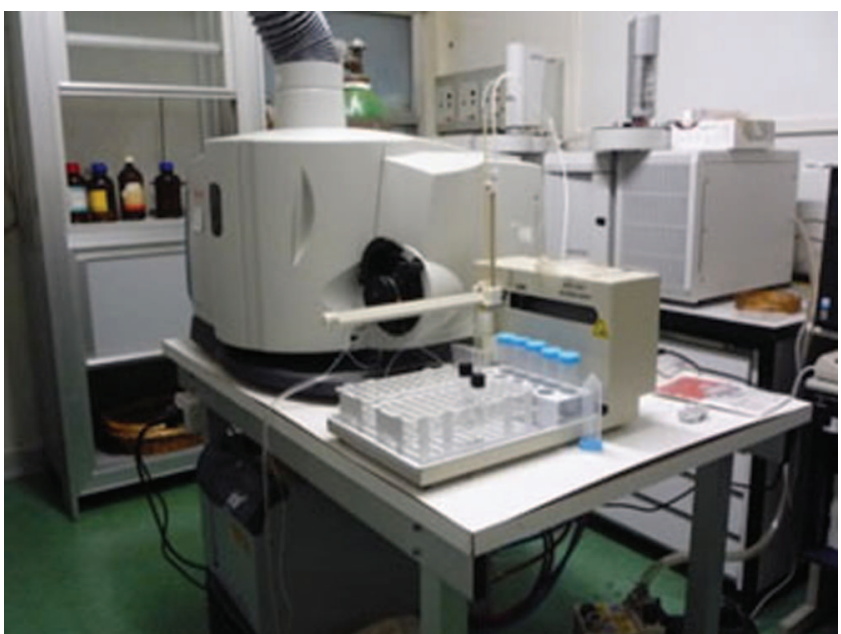

FIG. 13. FT-IR validation instrumentation (general view).

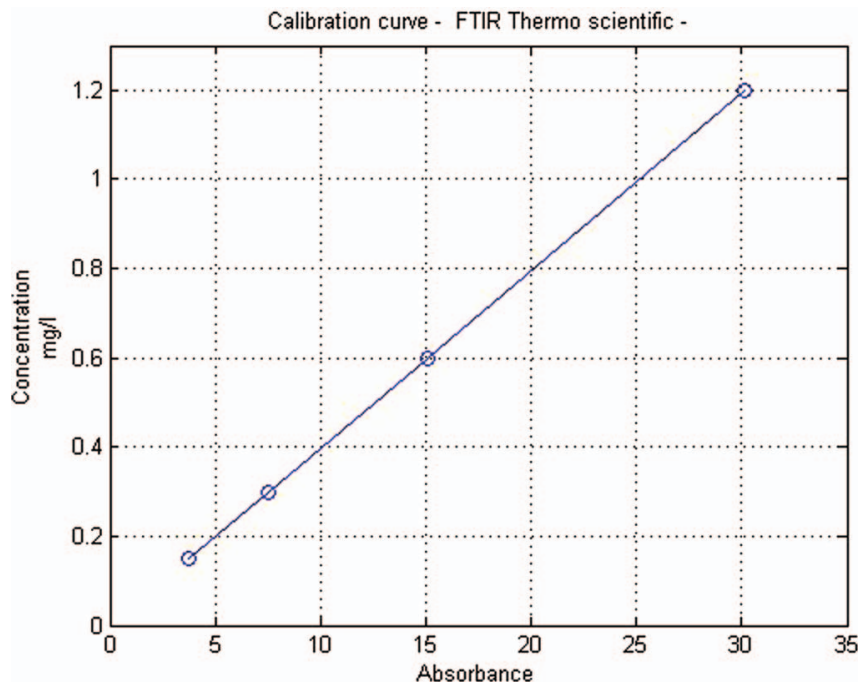

FIG. 14. Calibration curve of new measured data by FT-IR Thermo scientific: $\mathrm{x}$-axis and $\mathrm{y}$-axis display absorbance and concentration respectively.

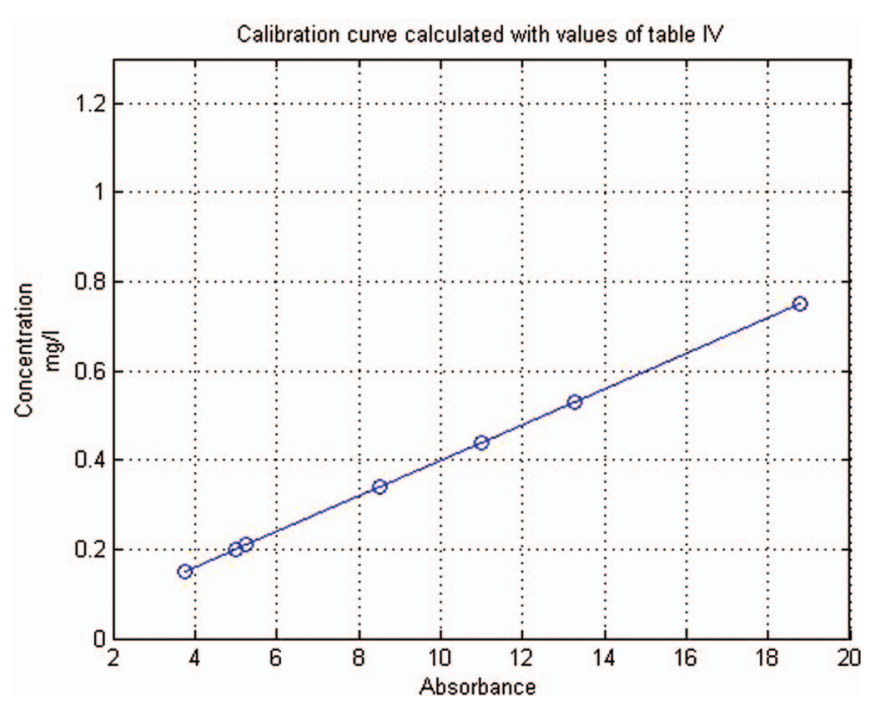

FIG. 15. Calibration curve calculated with values of Table IV: $x$-axis and $\mathrm{y}$-axis display absorbance and concentration respectively. 
TABLE IV. Absorbance calculated with FTIR Thermo scientific.

\begin{tabular}{lcr}
\hline \hline Samples & Concentration $(\mathrm{mg} / \mathrm{l})$ & Absorbance \\
\hline S1 & 0.15 & 3.77 \\
S2 & 0.3 & 7.54 \\
S3 & 0.6 & 15.08 \\
S4 & 1.2 & 30.16 \\
\hline \hline
\end{tabular}

detection of microparticles is easy to observe: by increasing the vibration frequency the absorption becomes more evident (see Figs. 11 and 12) and when the vibration is stopped the spectrum returns to the initial configuration by proving that, even in presence of vibrations, the proposed system is able to detect hydrocarbons contained in water.

\section{VALIDATION MEASUREMENTS}

Using samples belonging to the same kind of water, collected in a gourd, and a new generation instrumentation FT-IR by Thermo Scientific, ${ }^{15}$ it is possible to perform a further validation of our experimental measurements. In Fig. 13, a general view of validation instrumentation is shown. Thanks to the new instrumentation we obtain new measurements of concentrations, especially those regarding the absorbance of four samples included in the set used for this paper, as depicted in Table IV.

With this new measurements, we can trace a calibration curve, as indicated in Fig. 14. Using this curve we can find absorbance of every sample with concentration between value of S1 and S4, according to the plot of Fig. 15.

Using the following relationship:

$$
A=-\log _{10} T \rightarrow T=-10^{A}
$$

it is possible to find transmittance in laminar flow. To validate measurements it is necessary to compare experimental measurements with new ones. Tables V and VI report a comparison between new measurements (absorbance 1 and transmittance 1) and experimental ones (absorbance 2 and transmittance 2). In the effort to be clear, we sentence that absorbance 1 and transmittance 1 are calculated using values obtained from FT-IR Thermo scientific while absorbance 2 and transmittance 2 are computed using experimental value. The deviation is pointed out in Table VII.

TABLE V. Comparison between absorbance values calculated with FT-IR Thermo scientific and experimental values.

\begin{tabular}{lcccc}
\hline \hline Label & Concentration (mg/l) & Absorbance (1) & Absorbance (2) & deviation \\
\hline 563 & 0.2 & 5.01 & 5.02 & 0.01 \\
565 & 0.15 & 3.76 & 3.77 & 0.01 \\
566 & 0.21 & 5.26 & 5.27 & 0.01 \\
570 & 0.34 & 8.52 & 8.54 & 0.02 \\
572 & 0.44 & 11.02 & 11.05 & 0.03 \\
575 & 0.53 & 13.28 & 13.32 & 0.04 \\
576 & 0.75 & 18.8 & 18.85 & 0.05 \\
\hline \hline
\end{tabular}

TABLE VI. Comparison between transmittance values calculated with FTIR Thermo scientific and experimental values.

\begin{tabular}{|c|c|c|c|c|}
\hline Label & $\begin{array}{c}\text { Concentration } \\
(\mathrm{mg} / \mathrm{l})\end{array}$ & $\begin{array}{c}\text { Transmittance } \\
\text { (1) }\end{array}$ & $\begin{array}{c}\text { Transmittance } \\
\text { (2) }\end{array}$ & Delta \\
\hline 563 & 0.2 & 102329.3 & 104712.9 & 2383.56 \\
\hline 565 & 0.15 & 5623.41 & 5888.43 & 265.02 \\
\hline 566 & 0.21 & 181970.08 & 186208.71 & 4238.63 \\
\hline 570 & 0.34 & 331131121.48 & 346736850.45 & $1.6 \times 10^{+7}$ \\
\hline 572 & 0.44 & $1.04771 \times 10^{+11}$ & $1.122 \times 10^{+11}$ & $7.5 \times 10^{+9}$ \\
\hline 575 & 0.53 & $1.9055 \times 10^{+13}$ & $2.0893 \times 10^{+13}$ & $1.8 \times 10^{+12}$ \\
\hline 576 & 0.75 & $6.30957 \times 10^{+18}$ & $7.07946 \times 10^{+18}$ & $7.7 \times 10^{+17}$ \\
\hline
\end{tabular}

\section{SUMMARY AND FINAL OUTLOOK}

An experimental measurement system for IR spectroscopic characterization of water flow has been presented in this paper. It is considered cheaper with respect to the cost of the technology used to perform chemical analysis ${ }^{16}$ as per the description carried out for both instrumentation used at upstream and downstream of the proposed experimental system. Even if we operate at IR, no interferometer is used to obtain the foreseen results. It is important to sentence that the above results that consist in an alignment among the spectrometer, our experimental system and the FT-IR is due to the fact that the characterization has been performed in low temperature; that is, in the range between $15^{\circ} \mathrm{C}$ and $30^{\circ} \mathrm{C}$ for the sample. It is known that in many applications of midand far-infrared spectroscopy, interesting information can be obtained by reducing the temperature sample. In particular, temperature reduction has been suggested as a useful method for distinguishing between intra-molecular vibrations and lattices modes, since generally the frequency of lattice bands increases on cooling the sample while the absorption frequency of fundamental modes is usually unaffected. In general, the water emissions from industrial plants must happen in low temperature. If they take place in temperature greater than $22-25^{\circ} \mathrm{C}$, authorities impose a cooling system or an artificial canal so that the water must be spontaneously cooled. Warmed water, especially with pollutants is a worst combination that can destroy irreversible environmental matrices. That is the main reason of our proposal which is very simple to realize with respect to others ${ }^{17,18}$ since it is possible to perform online measurements. The proposed experimental system may be intended as an alert or a preliminary step for online monitoring. To prevent damages and disturbance

TABLE VII. Deviation computing.

\begin{tabular}{lccc}
\hline \hline Label & $\begin{array}{c}\text { Concentration } \\
(\mathrm{mg} / \mathrm{l})\end{array}$ & $\begin{array}{c}\text { Absorbance } \\
\text { deviation }(\%)\end{array}$ & $\begin{array}{c}\text { Transmittance } \\
\text { deviation (\%) }\end{array}$ \\
\hline 563 & 0.2 & 0.20 & 2.28 \\
565 & 0.15 & 0.27 & 4.5 \\
566 & 0.21 & 0.19 & 2.28 \\
570 & 0.34 & 0.23 & 4.5 \\
572 & 0.44 & 0.27 & 6.67 \\
575 & 0.53 & 0.30 & 8.8 \\
576 & 0.75 & 0.27 & 10.87 \\
\hline \hline
\end{tabular}


during analysis, the samples are riddled, with an ad hoc subsystem, as it is done in any analytical instrumentation in order to remove mineral particles.

\section{ACKNOWLEDGMENTS}

The authors gracefully thank the Italian National Institute for Micro-pollutants (INCA)-University of Salento, for its technical support and cooperation. The authors also thank Stante laboratory of Taranto and Dr A. Massaro for useful advices.

${ }^{1}$ United Nations Educational, Scientific and Cultural Organization (UNESCO), UNESCO/Berghahn Book, 2009.

${ }^{2}$ T. G. Huntington, J. Hydrol. 319, 83 (2006).

${ }^{3}$ T. Oki and S. Kanae, Science 313, 1068 (2006).

${ }^{4}$ United States Geological Survey (USGS), USGS Circ. 1, 1139 (1998).

${ }^{5}$ R. P. Schwarzenbach, B. I. Escher, K. Fenner, T. B. Hofstetter, C. A. Johnson, U. von Gunten, and B. Werhli, Science 313, 1072 (2006).
${ }^{6}$ J. R. V. Zaneveld, M. S. Twardowki, K. S. Shifrin, W. S. Pegau, E. Boss, and I. Zolotov, in Proceedings of Ocean Optics XVI, Santa Fe, NM, 2002.

${ }^{7}$ T. A. O'Bree, Ph.D. dissertation (RMIT University, Victoria, Australia, 2007), p. 7.

${ }^{8}$ K. Okada, M. Aoyagi, and S. Iwata, J. Quant. Spectrosc. Radiat. Transf. 72, 813 (2002).

${ }^{9}$ C. F. Bohren and D. R. Huffman, Absorption and scattering of light by small particles (Wiley, 1983).

${ }^{10}$ R. P. Schwarzenbach, T. Egli, T. B. Hofstetter, U. von Gunten, and B. Wehrli, Annu. Rev. Environ. Resour. 35, 109 (2010).

${ }^{11}$ A. Massaro, F. Spano, D. Caratelli, A. Yarovoy, R. Cingolani, and A. Athanassiou, Int. J. Meas. Technol. Instrum. Eng. 1, 55 (2011).

${ }^{12}$ A. Massaro, Transworld Research Network, 2001.

${ }^{13}$ F. Spano, A. Massaro, R. Cingolani, and A. Athanassiou, J. Microelectron. Eng. 88, 2763 (2011).

${ }^{14}$ See www.agilent.com for mass spectrometer and chromatographic gas issues.

${ }^{15}$ See www.thermoscientific.com for FT-IR instrumentation.

${ }^{16}$ F. Vogt, M. Tacke, M. Jakusch, and B. Mizaikoff, Anal. Chim. Acta 422, 187 (2000).

${ }^{17}$ A. Barth, Biochim. Biophys. Acta 1767, 1073 (2007).

${ }^{18}$ D. Jiao, A. A. Ergin, B. Shanker, E. Michielssen, and J. Jian-Ming, IEEE Trans. Antennas Propag. 50, 1192 (2002). 